\title{
Ventilation Coefficients for Falling Ice Crystals in the Atmosphere at Low-Intermediate Reynolds Numbers
}

\author{
Wusheng Ji AND PAO K. WANG \\ Department of Atmospheric and Oceanic Sciences, University of Wisconsin-Madison, Madison, Wisconsin
}

(Manuscript received 20 November 1997, in final form 8 May 1998)

ABSTRACT

\begin{abstract}
The ventilation coefficients for columnar, hexagonal plate, and broad branch ice crystals falling in air are computed by first solving numerically the convective diffusion equation for water vapor density to obtain its profile around these ice crystals and then determining the total vapor flux on the surface of the crystal. The ratio of this flux to the flux on a stationary crystal gives the ventilation coefficient. The local flow velocity profiles around the falling crystals necessary for specifying the convective term in the convective diffusion equation were obtained previously by numerically solving the unsteady Navier-Stokes equations subject to appropriate crystal-shaped boundary conditions. Ventilation coefficients obtained in this way are illustrated as a function of the Schmidt and Reynolds numbers and are also fitted by empirical expressions. Applications of these ventilation coefficients are discussed.
\end{abstract}

\section{Introduction}

In order to determine the diffusional growth rate of cloud and precipitation particles, it is necessary to take into account the fact that these particles are moving relative to the air. This relative motion causes the air to flow around the crystal in a complicated way, which in turn influences the distribution of water vapor around the crystal. Since the diffusional growth rate of the particle is determined by the vapor density gradient, it is obviously influenced by the motion. This effect on the growth (or evaporation if the air is subsaturated) of cloud and precipitation particles is known as the ventilation effect. Due to this effect, a falling hydrometeor will grow (or evaporate) faster than when it is stationary relative to the air. This is due to the enhancement of the mean vapor density gradient around the hydrometeor. The magnitude of enhancement is given by a factor called the "mean ventilation coefficient" defined as (Pruppacher and Klett 1997)

$$
\bar{f}_{v}=\frac{(d m / d t)}{(d m / d t)_{0}},
$$

where the numerator and the denominator represent the growth rate of the hydrometeor with mass $m$ under moving and stationary conditions, respectively.

Corresponding author address: Dr. Pao K. Wang, Department of Atmospheric and Oceanic Sciences, University of Wisconsin-Madison, 1225 W. Dayton Street, Madison, WI 53706.

E-mail: pao@windy.meteor.wisc.edu
This paper is concerned with the ventilation effect on falling ice crystals in atmospheric clouds. Unlike the more thoroughly studied cases of water droplets (see Pruppacher and Klett 1997, chap. 13, for a review), there exists little information for ice crytstals. The mean ventilation coefficient can be either measured experimentally in the laboratory or computed theoretically. Experimental measurements, especially for ice crystals, require sophisticated equipment and are difficult to perform, and thus far only a few direct measurements have been done for ice spheres and hexagonal ice plates (Thorpe and Mason 1966), and some indirect information has been inferred from experimental studies of snow crystal growth (Takahashi et al. 1991). Theoretical computations of the ventilation coefficients have been carried out by Brenner (1963), who obtained analytical solutions of the convective diffusion equation for infinitely thin circular disks, and by Masliyah and Epstein (1971) and Pitter et al. (1974), who computed numerically the ventilation coefficients of thin oblate spheroids of various axis ratios used for approximating hexagonal plates.

The present paper reports results obtained by the authors also based on numerical techniques similar to Masliyah and Epstein (1971) and Pitter et al. (1974) but without using the thin oblate spheroid approximation for hexagonal plates. Instead, the true shape of hexagonal plates is used directly. In addition, the ventilation coefficients for falling columnar and broad-branch ice crystals are also computed. The three types of crystals considered in the present study are the same as those in Wang and Ji (1997; see their Fig. 1). The mathematics 
and physics of the problem and the numerical methods employed to obtain solutions are given in the following sections.

\section{Physics and mathematics}

The theoretical problem of determining the ventilation coefficients for falling ice crystals is basically a convection diffusion problem for water vapor around the crystals. The convective diffusion equation appropriate for this situation is

$$
\frac{\partial \rho_{v}}{\partial t}=D_{v} \nabla^{2} \rho_{v}-\mathbf{V} \cdot \nabla \rho_{v},
$$

where $\rho_{v}$ is vapor density, $D_{v}$ the diffusion coefficient of water vapor in air, and $\mathbf{V}$ the local air velocity vector. The boundary conditions are

$$
\begin{cases}\rho_{v}=\rho_{v s} & \text { at the surface of the crystal } \\ \rho_{v}=\rho_{v \infty} & \text { far away from the crystal, }\end{cases}
$$

where $\rho_{v s}$ and $\rho_{v \infty}$ are two constants representing the vapor density at the surface of and far away from the crystal, respectively. Unlike the case of spheres where the inner boundary can be easily written down as the surface where $r=a$ ( $a$ radius of the sphere), the surface of an ice crystal cannot be easily expressed mathematically. ${ }^{1}$ For the present study, the boundary conditions are specified numerically.

Equation (2) is written in dimensional form. To facilitate the numerical calculations, this equation is nondimensionalized by introducing the following nondimensional quantities:

$$
\left\{\begin{aligned}
\phi & =\frac{\rho_{v}-\rho_{v \infty}}{\rho_{v s}-\rho_{v \infty}} \\
x^{\prime} & =x / a \\
t^{\prime} & =t V_{\infty} / a \\
\mathbf{V}^{\prime} & =\mathbf{V} / V_{\infty} \\
N_{\mathrm{Pe}} & =2 a V_{\infty} / D_{v} .
\end{aligned}\right.
$$

All the left-hand side quantities are dimensionless. The variable $x$ (or $y, z$ ) is one of the three Cartesian coordinates, $t$ the time, $V_{\infty}$ the free stream velocity (or the air velocity far away from the ice crystal surface), $a$ the radius of the crystal (measured from the center to one of the furthest tip), and $N_{\mathrm{Pe}}$ the Peclet number. Using the new dimensionless variables, Eq. (2) becomes

\footnotetext{
${ }^{1}$ Wang and Denzer (1983) and Wang (1987, 1997) have found some simple expressions that can be used to represent the shape of some ice crystals, but they have not been applied to the present study. These expressions are mostly for two-dimensional shapes, while the present study requires three-dimensional expressions. New three-dimensional expressions are given in a coming paper.
}

TABLE 1. Reynolds numbers, dimensions, and capacitance of columnar ice crystals in the present study. The quantities are dimensionless.

\begin{tabular}{rccc}
\hline \hline$N_{\mathrm{Re}}$ & Diameter & Length & Capacitance \\
\hline 0.2 & 2.0 & 2.85 & 1.3628 \\
0.5 & 2.0 & 2.85 & 1.3628 \\
0.7 & 2.0 & 3.08 & 1.4054 \\
1.0 & 2.0 & 3.33 & 1.4535 \\
2.0 & 2.0 & 4.44 & 1.6511 \\
5.0 & 2.0 & 6.67 & 2.0151 \\
10.0 & 2.0 & 10.00 & 2.5067 \\
20.0 & 2.0 & 16.67 & 3.3959 \\
\hline
\end{tabular}

$$
\frac{\partial \phi}{\partial t}=\frac{2}{N_{\mathrm{Pe}}} \nabla^{2} \phi-\mathbf{V} \cdot \boldsymbol{\nabla} \phi,
$$

and the boundary conditions (3) become

$$
\begin{cases}\phi=1 & \text { at the surface of the crystal } \\ \phi=0 & \text { far away from the crystal. }\end{cases}
$$

Equations (5) and (6) are the nondimensional set of equations that are to be solved numerically. While the first (inner) boundary condition can be applied in a straightforward manner, some considerations have to be given before the second (outer) boundary condition can be implemented in the actual computations. The ideal theoretical outer boundary is normally put at $r \rightarrow \infty$, which is obviously impossible to do in a real numerical scheme on which the present study is based. Thus, some finite outer boundary surfaces have to be devised sufficiently far from the crystal to replace the ideal one.

There are additional considerations for setting the boundary conditions. First of all, the solution of the convective diffusion equation (2) requires the knowledge of local air velocity vector at each point of the numerical grid. In the present study these velocity vectors are obtained from the numerical solutions of the time-dependent Navier-Stokes equations for incompressible flow past ice crystals as reported recently by Wang and Ji (1997). Thus, it is necessary for consistency of precision that we use a numerical grid that is either the same or smaller than the one used in Wang and Ji (1997) for flow field calculations. We choose to use the same grid and hence the same boundary surfaces for the present study. The locations of the outer boundaries are given in Table 1 of Wang and Ji (1997).

Second, while the second condition in (6) can be applied at the upstream and lateral outer boundaries, it will encounter difficulty at the downstream outer boundary in the cases where the flows are unsteady. Due to the restriction of limited computing resource and hence the finite downstream boundary, the requirement of a constant $\phi$ at this distance is most likely unrealistic. Here we replace this condition by the following:

$$
\frac{\partial \phi}{\partial z}=0 .
$$


TABLE 2. Reynolds numbers, dimensions, and capacitance of hexagonal ice plates in the present study. The quantities are dimensionless.

\begin{tabular}{rccc}
\hline \hline \multicolumn{1}{c}{$N_{\mathrm{Re}}$} & Diameter & Thickness & Capacitance \\
\hline 1.0 & 2.0 & 0.2250 & 0.7298 \\
2.0 & 2.0 & 0.1770 & 0.6977 \\
10.0 & 2.0 & 0.1265 & 0.6639 \\
20.0 & 2.0 & 0.1034 & 0.6485 \\
35.0 & 2.0 & 0.0863 & 0.6371 \\
60.0 & 2.0 & 0.0725 & 0.6278 \\
90.0 & 2.0 & 0.0640 & 0.6221 \\
120.0 & 2.0 & 0.0576 & 0.6179 \\
\hline
\end{tabular}

This simply means that we require the $\phi$ field to be continuous at the downstream boundary surface. A similar condition for velocity had been used by the authors for solving the flow field problem and worked well (Wang and Ji 1997), and, as it turned out, so does this one.

Once the $\phi$ profile is determined, the growth rate of the falling ice crystal (without considering the coupling of latent heat released or consumed) can be calculated using

$$
\frac{d m}{d t}=-\oint_{s} D_{v} \boldsymbol{\nabla} \phi \cdot d \mathbf{S},
$$

where the integration is to be carried out over the surface of the ice crystal $S$. On the other hand, the growth rate of a stationary ice crystal is given by the classic electrostatic analog:

$$
\left(\frac{d m}{d t}\right)_{0}=-4 \pi C D_{v}\left(\rho_{v s}-\rho_{v \infty}\right),
$$

where $C$ is the capacitance of the ice crystal (see Pruppacher and Klett 1997). Hence in order to calculate the growth rates of stationary ice crystals, it is necessary to have the values of their capacitance.

For columnar ice crystals, which are approximated by finite circular cylinders in this study, we used Smythe's (1956, 1962) and Wang et al.'s (1985) formulation to calculate the values of capacitance. The dimensions and the capacitance of the cylinders so determined in this study are given in Table 1. For the capacitance of hexagonal plates we used the formulation of McDonald (1963), who measured the capacitance of various conductors cut in the shape of snow crystals. The theoretical values of $C$ for hexagonal plates of small thickness may be written as

$$
C=\frac{2 \times 0.91 a}{\pi}\left(\frac{1+\Delta S}{S}\right),
$$

where $a$ is the radius (measured from the center to one of the edges), $S$ the area of the basal plane, and $\Delta S$ the area difference between the hexagon and a circle with the same radius. The dimensions and the capacitance of
TABLE 3. Reynolds numbers and dimensions of broad-branch ice crystals in the present study. The quantities are dimensionless.

\begin{tabular}{rcc}
\hline \hline$N_{\mathrm{Re}}$ & Diameter & Thickness \\
\hline 1.0 & 2.0 & 0.15 \\
2.0 & 2.0 & 0.14 \\
10.0 & 2.0 & 0.0914 \\
20.0 & 2.0 & 0.080 \\
35.0 & 2.0 & 0.0667 \\
60.0 & 2.0 & 0.060 \\
90.0 & 2.0 & 0.052 \\
120.0 & 2.0 & 0.047 \\
\hline
\end{tabular}

the hexagonal plates calculated according to this formula are given in Table 2 .

Unfortunately, there are no accurate values of either measured or theoretically calculated capacitance available for broad-branch crystals. Thus in this case, we compute the growth rate of stationary broad-branch crystals directly by numerically solving the (nonconvective) diffusion equation first and then using (1) to determine the ventilation coefficient. The dimensions of the broad-branch crystals involved in the present study are given in Table 3.

When the flow is unsteady, the computed ventilation coefficient will differ a little bit at different time steps. The final value of the coefficient is taken as the average value over one eddy shedding cycle. Since the eddy shedding occurs mainly in the downstream, its influence on the coefficient is not very large, typically smaller than $10 \%$.

The numerical scheme (including the grid and the iteration and interpolation techniques) used in the present study is identical to that of Wang and Ji (1997) for obtaining the flow fields around falling crystals. Readers are referred to this article for details.

\section{Results and discussion}

As mentioned previously, the ventilation coefficients of three different ice crystal habits of various dimensions (as listed in Tables 1, 2, and 3) are computed according to the method described in section 2 . The atmospheric pressure and temperature are assumed to be $P=800 \mathrm{hPa}$ and $T=-8^{\circ} \mathrm{C}$. It may seem unnecessary to define $P$ and $T$ for the calculations of the ventilation coefficients as they are controlled strictly by hydrodynamics. But the pressure and temperature do affect the values of several nondimensional characteristic numbers to be introduced below and, therefore, need to be specified.

Figure 1 shows an example of vapor density field around a stationary columnar ice crystal. The field obviously possesses symmetry with respect to the crystal since no motion is involved here. Similar distributions can also be seen in Wang et al. (1985) for potential fields around finite cylinders. Once the motion is introduced, however, the symmetry disappears, and the resulting vapor density fields show enhanced gradients in 
(a)

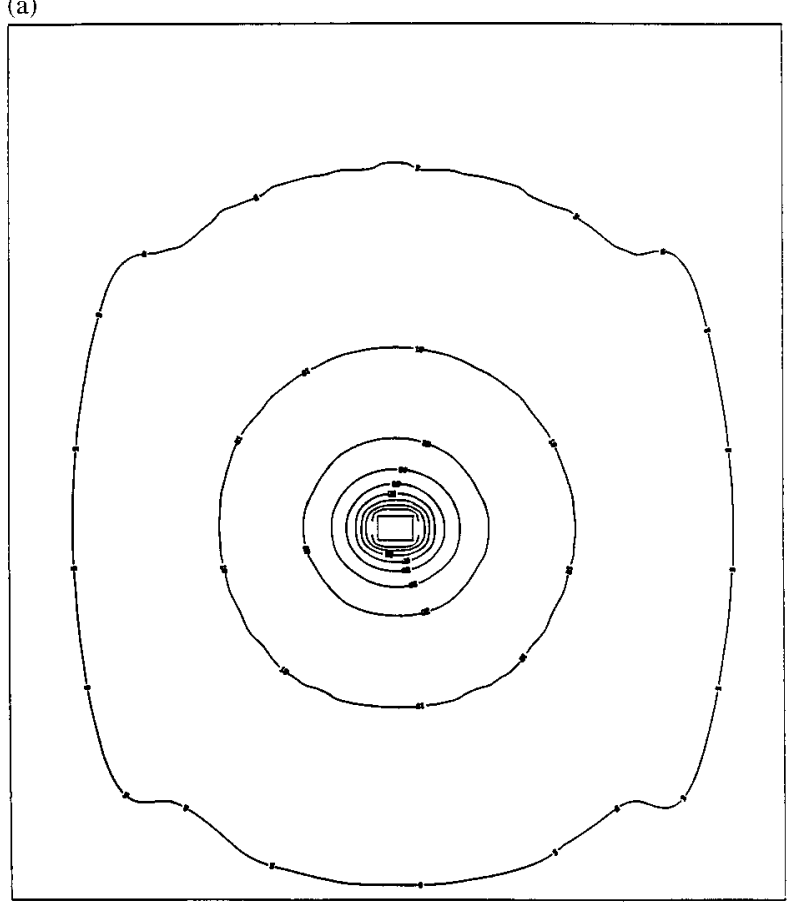

(b)

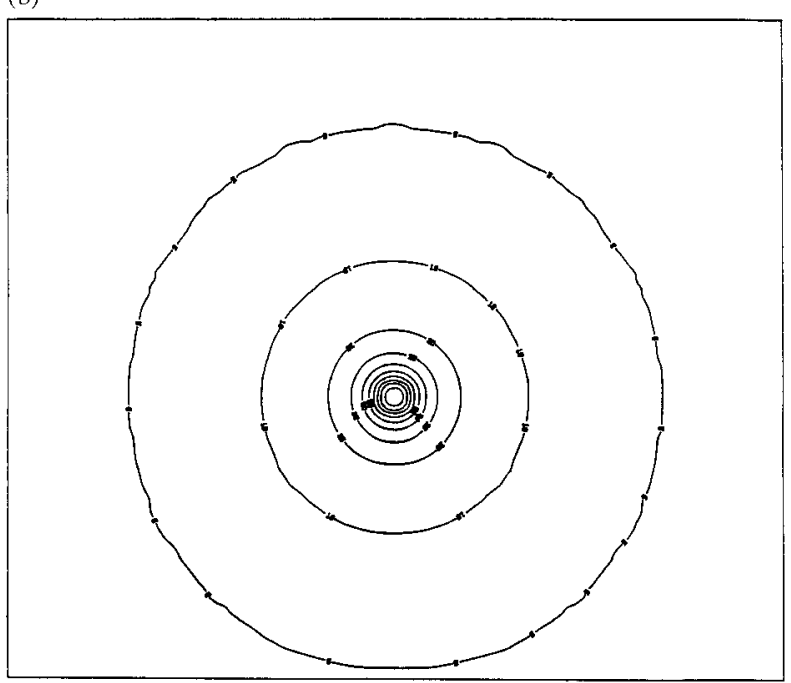

FIG. 1. Water vapor density distribution around a stationary ice column at $N_{\mathrm{Re}}=0.2$. (a) Length view. (b) End view. The vapor density field is normalized so that the density is 100 on the surface. The contour levels are (from outside) 2, 10, 20, 30, 40, 50, 60, 70, 80, and 100 (surface). The contour level 90 is not shown to avoid overcrowding of curves.

the upstream and relaxed gradients in the downstream, as illustrated by Figs. 2 and 3 for columnar crystals falling at Reynolds numbers 2 and 10, respectively. Comparison of these two figures clearly shows that the higher the Reynolds number, the more pronounced the asymmetry of the vapor density fields and the greater the enhancement of the upstream gradients. Figure 4

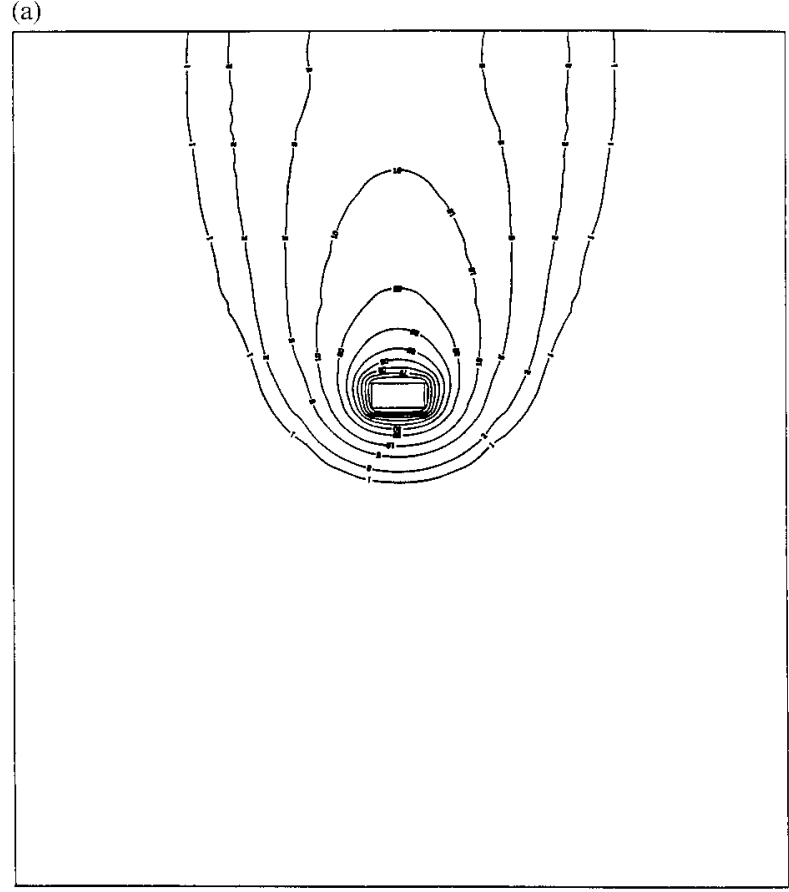

(b)

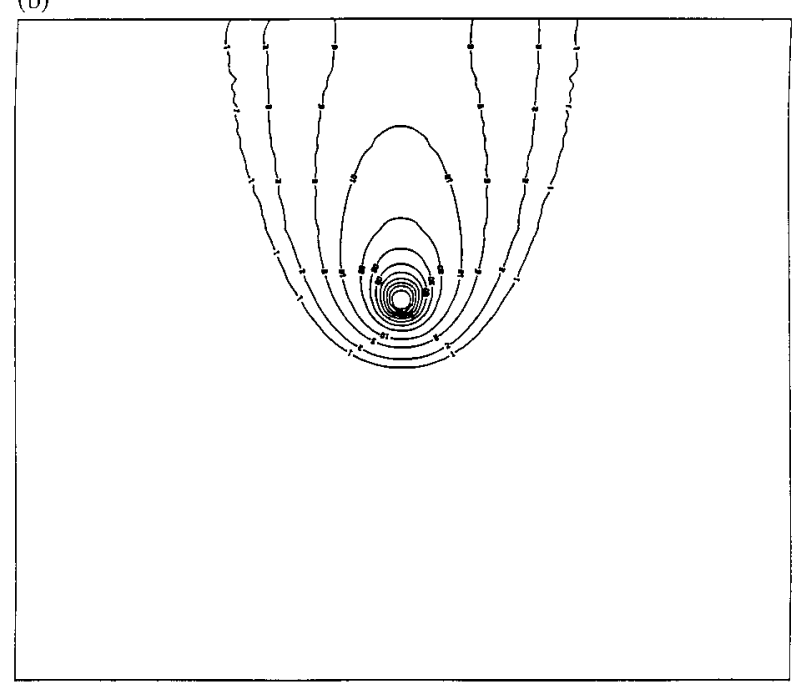

FIG. 2. Water vapor density distribution around a falling ice column at $N_{\mathrm{Re}}=2.0$. (a) Length view. (b) End view. The contour levels are (from outside) 1, 2, 5, 10, 20, 30, 40, 50, 60, 70, 80, and 100 (surface). The contour level 90 is not shown to avoid overcrowding of curves.

shows the vapor density fields around falling broadbranch crystals at Reynolds number 2 . The main features are essentially the same as the two previous figures.

For higher Reynolds number cases where the flow fields become unsteady [see Wang and Ji (1997) for examples of these flow fields], the vapor density fields also become unsteady, but the main feature of front enhancement and rear relaxation of vapor density fields remain the same.

The mean ventilation coefficient is then calculated 


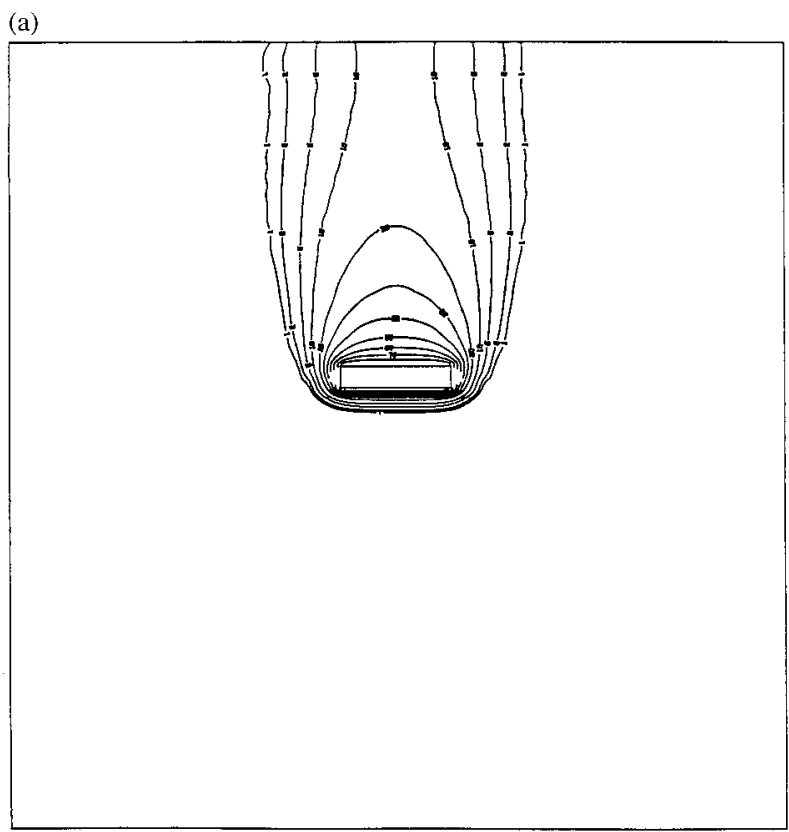

(b)

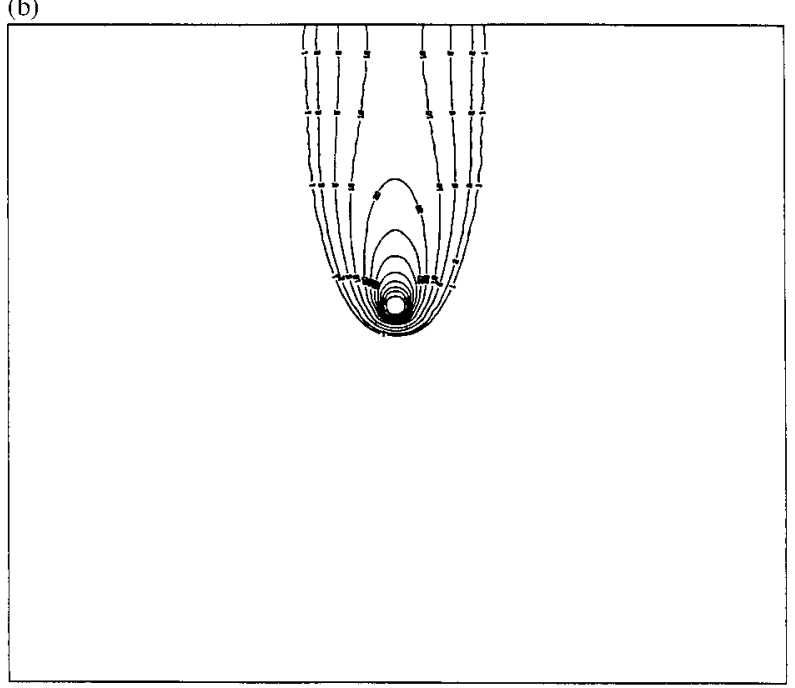

FIG. 3. Water vapor density distribution around a falling ice column at $N_{\mathrm{Re}}=10.0$. (a) Length view. (b) End view. The contour levels are (from outside) 1, 2, 5, 10, 20, 30, 40, 50, 60, 70, 80, and 100 (surface). The contour level 90 is not shown to avoid overcrowding of curves.

using (8), (9), and (1), and the results are summarized in Fig. 5. The horizontal axis is a dimensionless number, $X$, defined as

$$
X=\left(N_{\mathrm{Scv}}\right)^{1 / 3}\left(N_{\mathrm{Re}}\right)^{1 / 2},
$$

where $N_{\mathrm{Scv}}$ is the Schmidt number of water vapor $\left(N_{\mathrm{Sc}}\right.$ air kinematic viscosity/water vapor diffusivity), and $N_{\mathrm{Re}}$ the Reynolds number of the falling ice crystal. It is seen that the functional dependence of $\bar{f}_{v}$ on $X$ is similar to that found by previous investigators [see Pitter et al. (1974) for a summary]. The results of Thorpe and Ma- son (1966) and Pitter et al. (1974) are also plotted for comparison. Pitter et al. (1974) used thin oblate spheroids of axis ratio 0.05 to approximate hexagonal plates and determined their flow fields and ventilation coefficients. The appropriate corresponding case in our results is the hexagonal plate at $X \sim 3.85$. It is seen here that their result is greater than the present result by about $10 \%$. The difference is probably due to the different aspect ratio and the somewhat different shape of cross section of the crystals. On the other hand, their results are close to the present results for broad-branch crystals. Thorpe and Mason's (1966) experimental results are greater than both our and Pitter et al.'s results but are close to that obtained by Masliyah and Epstein's (1971) numerical results for oblate spheroids of axis ratio 0.2. Apparently the axis ratio is one parameter that must be considered in characterizing the ventilation coefficients. Unfortunately, there are no experimental measurements that we are aware of at present for verifying other results.

The three curves in Fig. 5 representing the present results can be fitted by the following empirical expressions:

$$
\begin{aligned}
\bar{f}_{v}= & 1.0-0.00668(X / 4)+2.39402(X / 4)^{2} \\
& +0.73409(X / 4)^{3}-0.73911(X / 4)^{4}
\end{aligned}
$$

for columnar ice crystals of $0.2 \leq N_{\mathrm{Re}} \leq 20$;

$$
\begin{aligned}
\bar{f}_{v}= & 1.0-0.60420(X / 10)+2.79820(X / 10)^{2} \\
& +0.31933(X / 10)^{3}-0.06247(X / 10)^{4}
\end{aligned}
$$

for simple hexagonal plates of $1.0 \leq N_{\mathrm{Re}} \leq 120$; and

$$
\bar{f}_{v}=1.0+0.35463(X / 10)+3.55338(X / 10)^{2}
$$

for broad-branch crystals of $1.0 \leq N_{\mathrm{Re}} \leq 120$.

In the present study, $N_{\mathrm{Sc}}$ is held at a constant value $(=0.63)$, so what the figure shows is essentially the variation of the ventilation coefficient with the Reynolds number. Figure 6 shows this relation. Here we see that the dependence is nearly linear. However, while it is unlikely that the linear trend in the columnar case can be continued much further, it is likely that the slope will become smaller at higher Reynolds number.

Figure 6 also shows that, at a fixed Reynolds number, the columnar ice crystal has the highest ventilation coefficient among the three habits. This is probably due to the fact that the characteristic dimension of the column used in defining its Reynolds number is its radius instead of its length, so a columnar crystal of a small Reynolds number actually represents a long crystal and hence high fall velocity. This high velocity (higher than both the hexagonal plate and broad-branch crystal at the same $N_{\mathrm{Re}}$ ) is the main reason for its higher ventilation coefficient. It is less obvious why the ventilation coefficient of a broad-branch crystal is higher than a hexagonal plate at the same $N_{\mathrm{Re}}$, but again this may be explained by looking at the dimension of the crystals. 
(a)

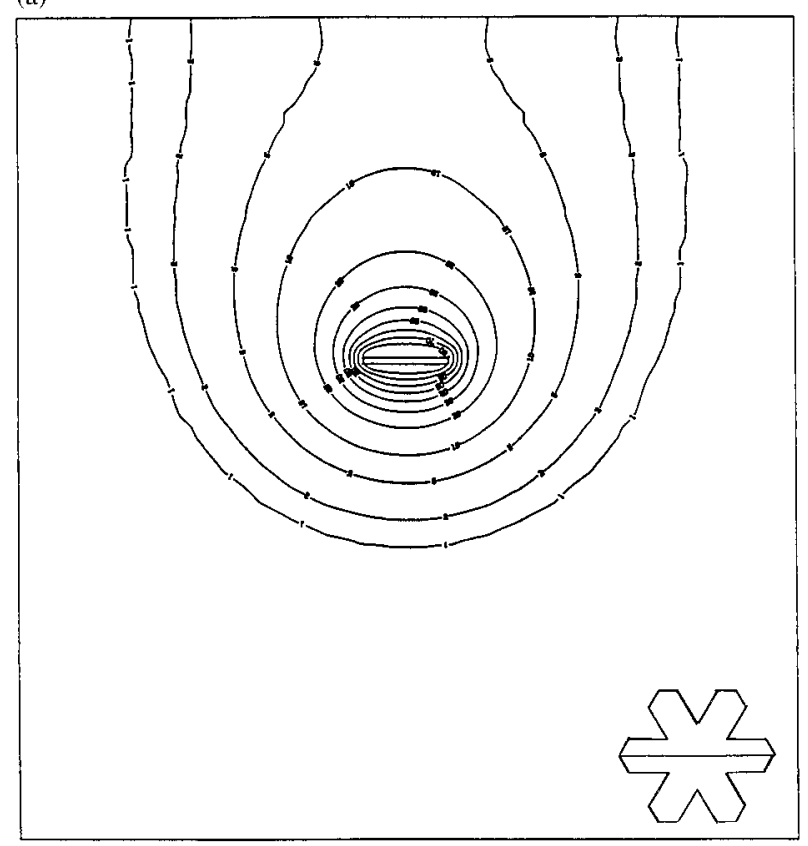

(b)

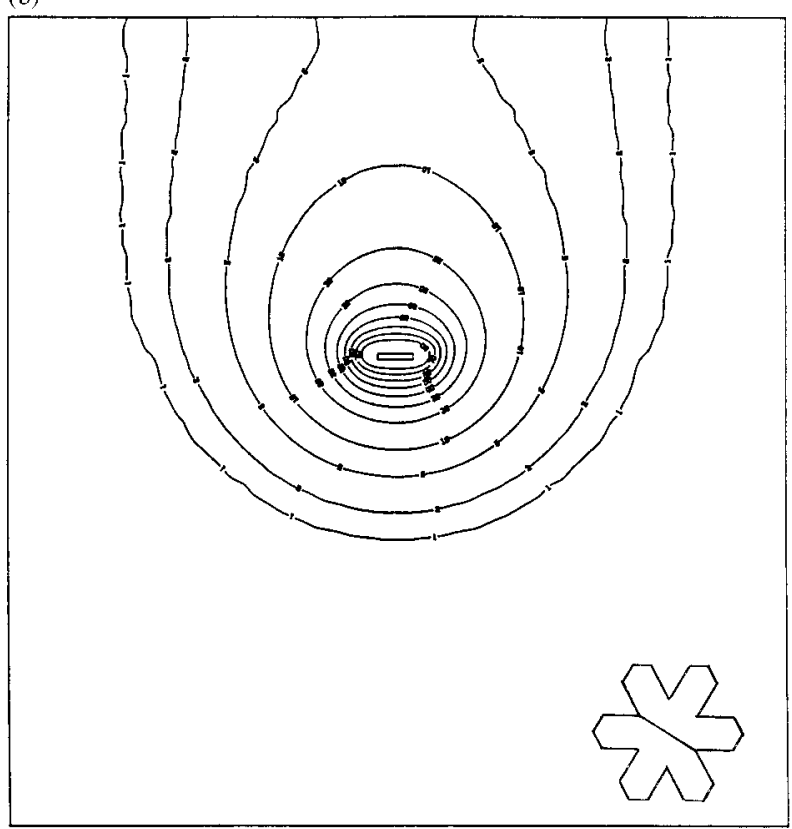

FIG. 4. Water vapor density distribution around a falling broad-branch crystal at $N_{\mathrm{Re}}=2.0$. (a) Central cross-sectional view. (b) Diagonal cross-sectional view. The contour levels are (from outside): 1, 2, 5, 10, 20, 30, 40, 50, 60, 70, 80, and 100 (surface). The contour level 90 is not shown to avoid over-crowding of curves.

Due the more skeletal structure of the broad-branch crystal, its bulk density is smaller than a simple hexagonal plate. Hence, at the same Reynolds number, the dimension of the broad-branch crystal is larger than a

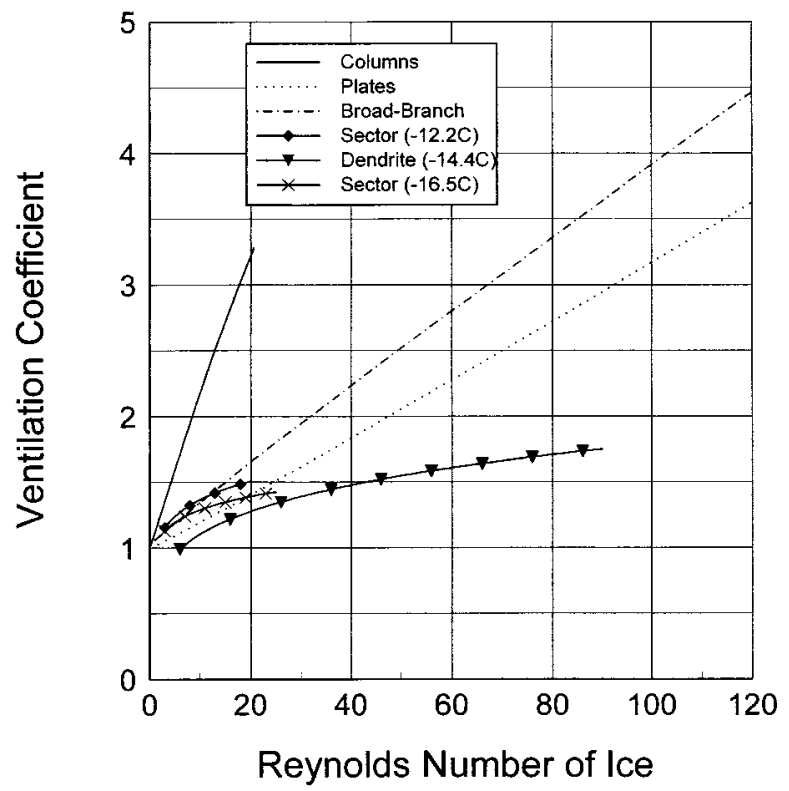

FIG. 5. Computed mean ventilation coefficients as a function of the dimensionless parameter $X$ defined in Eq. (11). Experimental results of Thorpe and Mason (1966) and numerical results of Pitter et al. (1974) are also plotted for comparison. hexagonal plate, and the surface area that can be subjected to the ventilation effect is also greater.

Takahashi et al. (1991) performed wind tunnel studies of the diffusional growth of free falling snow crystals between $-3^{\circ}$ and $-23^{\circ} \mathrm{C}$. They deduced some ventilation coefficients from the growth rates and their results (taken from their empirical relations) are plotted in Fig. 6 for comparison. Since the habits and aspect ratios of their crystals are different from the present, it is impossible to make exact comparisons. However, the magnitudes of the ventilation coefficients for their two sector cases are rather close to the present results for $N_{\mathrm{Re}}$ between 0 and 20, although the functional dependence appears to be different. The different functional dependence may be because the habit had changed significantly during the growth in their experiments, and the curve represents the coefficients for a particular continuously growing crystal rather than that for a crystal of fixed habit. The coefficients for their dendrite case are smaller than all other cases presented in Fig. 6, which seems to be intuitively expected since the open structure of dendrites would make them to fall slower than other habits and hence smaller $\bar{f}_{v}$.

\section{Application to heat ventilation problem}

Although the above ventilation calculations were based on the formulation of water vapor diffusion, which is a mass transfer process, the results are also applicable to the ventilation of heat for the same falling 


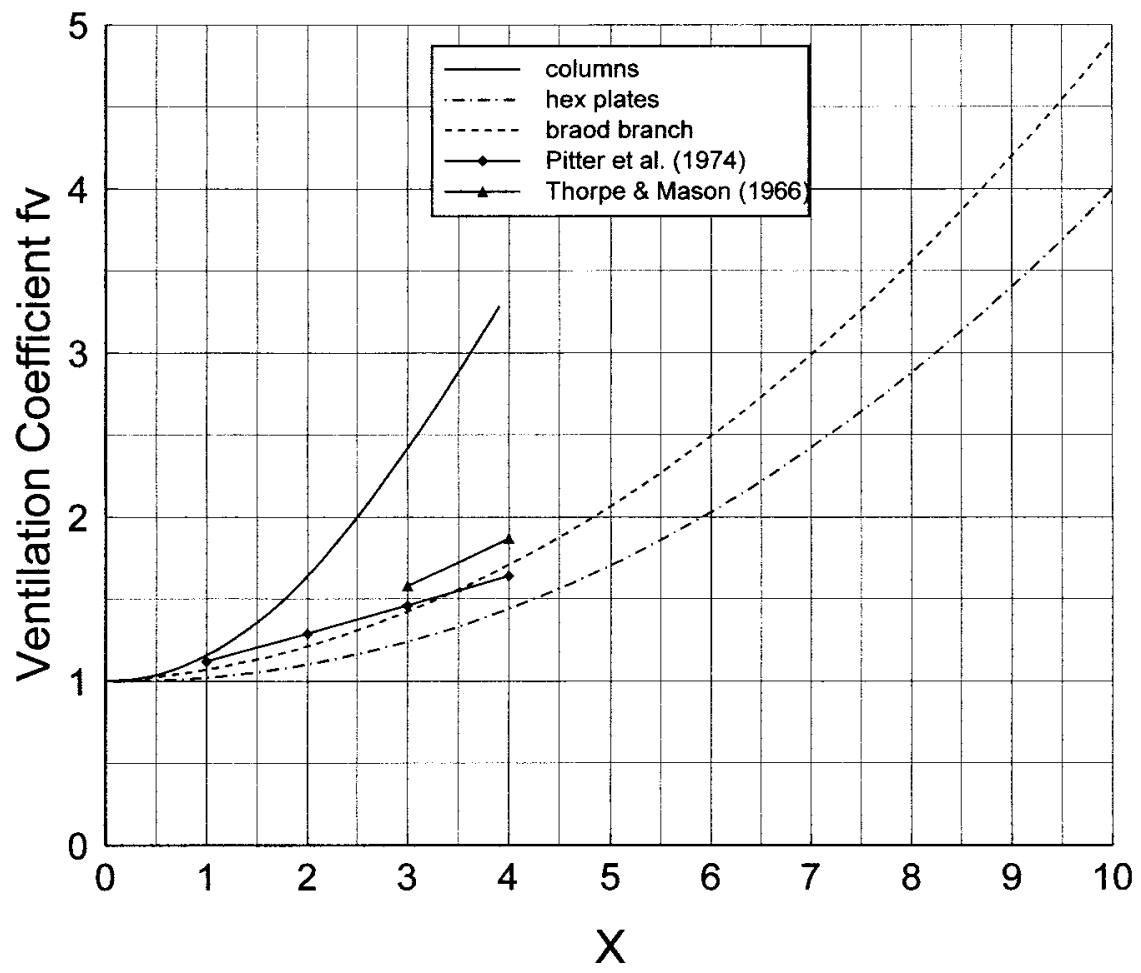

FIG. 6. Computed mean ventilation coefficients as a function of the Reynolds number of the falling ice crystal assuming the Schmidt number $=0.63$.

ice crystals since the mathematical equations involved (including the boundary conditions) are entirely the same except that the vapor density is replaced by temperature and the vapor diffusivity is replaced by the thermal conductivity of air. In the calculations of the diffusional growth rates of ice crystals, where the heat diffusion is coupled by vapor diffusion, it is adequate to set $\bar{f}_{v}=\bar{f}_{h}$, where the latter represents the mean ventilation coefficient of heat. Detailed discussions on the equivalence of these two phenomena and the coupling of them in determining the diffusional growth rates of ice crystals are given by Pruppacher and Klett (1997).

\section{Conclusions}

In the above, we have shown the calculations of ventilation coefficients of falling ice crystals of columnar, hexagonal plate, and broad-branch habits based on the convective diffusion theory of water vapor. The convective part of the mass transfer was computed using the detailed flow fields, computed by solving numerically the corresponding Navier-Stokes equations for the falling ice crystals. The result shows that the ventilation effect can be significant for the diffusional growth of ice crystals. A falling ice crystal may grow at a rate several times faster than a stationary one. Similarly, the heating or cooling due to the falling ice crystal's growth or evaporation can also be several times as large as a stationary one. Clearly both effects have a significant impact on the evolution of clouds that contain ice crystals, especially the cirrus clouds, where nearly all particles are ice crystals.

Acknowledgments. This work is partially supported by U.S. NSF Grant ATM-9314465, ATM-9633424, and ATM-9714158 to the University of Wisconsin-Madison. PKW would like to thank the Alexander von Humboldt Foundation of Germany for the Senior Research Humboldt Award he received and Johnson Wax Company of Racine, Wisconsin, for a distinguished fellowship to his research group that also contributed to this work.

\section{REFERENCES}

Brenner, H., 1963: Forced convection heat and mass transfer at small Peclect numbers from a particle of arbitrary shape. Chem. Eng. Sci., 18, 109-122.

Masliyah, J. H., and N. Epstein, 1971: Numerical study of steady flow past spheroids. J. Fluid Mech., 44, 493-512.

McDonald, J. E., 1963: Use of electrostatic analogy in studies of ice crystal growth. Z. Angew. Math. Phys., 14, 610.

Pitter, R. L., H. R. Pruppacher, and A. E. Hamielec, 1974: A numerical study of the effect of forced convection on mass tranport from a thin oblate spheroid of ice in air. J. Atmos. Sci., 31, 10581066.

Pruppacher, H. R., and J. D. Klett, 1997: Microphysics of Clouds and Precipitation. Kluwer Academic, 954 pp. 
Smythe, W. R., 1956: Charged right circular cylinders. J. Appl. Phys., 27, 917-920.

, 1962: Charged right circular cylinders. J. Appl. Phys., 33, 29662967.

Takahashi, T., T. Endoh, G. Wakahama, and N. Fukuta, 1991: Vapor diffusional growth of free falling snow crystals between -3 and $-23^{\circ}$ C. J. Meteor. Soc. Japan, 69, 15-30.

Thorpe, A. D., and B. J. Mason, 1966: The evaporation of ice spheres and ice crystals. Br. J. Appl. Phys., 17, 541-548.

Wang, P. K., 1987: Two-dimensional characterization of polygonally symmetric particles. J. Colloid Interface Sci., 117, 271-281.
_ 1997 : Characterization of ice crystals in clouds by simple mathematical expressions based on successive modification of simple shapes. J. Atmos. Sci., 54, 2035-2041.

— of plane hexagonal snow crystals. J. Atmos. Sci., 40, 1024-1028.

- and W. Ji., 1997: Numerical simulation of three-dimensional unsteady flow past ice crystals. J. Atmos. Sci., 54, 2261-2274.

, C. H. Chuang, and N. L. Miller, 1985: Electrostatic, temperature, and vapor density fields surrounding stationary columnar ice crystals. J. Atmos. Sci., 42, 2371-2379. 